\title{
Type 2 diabetes associated variants of $K C N Q 1$ strongly confer the risk of cardiovascular disease among the Saudi Arabian population
}

\author{
Maha S. Al-Shammari ${ }^{1}$, Rhaya Al-Ali ${ }^{1}$, Nader Al-Balawi ${ }^{1}$, Mansour S. Al-Enazi ${ }^{1}$, Ali A. Al-Muraikhi ${ }^{1}$, Fadi \\ N. Busaleh ${ }^{1}$, Ali S. Al-Sahwan ${ }^{1}$, Abdulmohsen Al-Elq ${ }^{2}$, Awatif N. Al-Nafaie ${ }^{2}$, Jesu Francis Borgio ${ }^{3}$, Sayed \\ AbdulAzeez ${ }^{3}$, Amein Al-Ali ${ }^{1}$ and Sadananda Acharya ${ }^{3 *}$ \\ ${ }^{1}$ College of Medicine, University of Dammam, Kingdom of Saudi Arabia. \\ ${ }^{2}$ King Fahd Hospital of the University, University of Dammam, Dammam, Kingdom of Saudi Arabia. \\ ${ }^{3}$ Institute for Research and Medical Consultations (IRMC), University of Dammam, Kingdom of Saudi \\ Arabia.
}

\begin{abstract}
Genome-wide association studies have identified several loci associated with an increased risk for cardiovascular disease (CVD) and type 2 diabetes (T2D). Polymorphisms within the KCNQ1 (potassium voltage-gated channel, KQT-like subfamily, member 1) gene are consistently associated with T2D in a number of populations. The current study was undertaken to evaluate the association of 3 polymorphisms of KCNQ1 (rs2237892, rs151290 and rs2237895) with T2D and/or CVD. Patients diagnosed with either T2D (320 patients), CVD (250 patients) or both (60 patients) and 516 healthy controls were genotyped by TaqMan assay run on a real time PCR thermocycler. A statistically significant association was found for SNPs rs $151290(\mathrm{OR}=1.76 ; 95 \% \mathrm{Cl}=1.02-3.05 ; \mathrm{p}=0.0435)$ and rs2237895 $(\mathrm{OR}=2.49 ; 95 \% \mathrm{Cl}=1.72-3.61 ; \mathrm{p}<0.0001)$ with CVD. SNP rs $151290(\mathrm{OR}=7.43 ; 95 \% \mathrm{Cl}=1.00-55.22 ; \mathrm{p}$ $=0.0499$ ) showed a strong association in patients with both T2D and CVD. None of the SNPs showed any significant association with T2D. Haploview analysis showed that the ACC (rs151290, rs2237892 and rs2237895) haplotype is the most significant risk allele combination for CVD, while CCA is the most significant risk haplotype for co-morbidity with T2D. KCNQ1 polymorphism at SNPs rs151290 and rs2237895 is strongly associated with CVD in this population, but presented no association with T2D.
\end{abstract}

Keywords: : T2D, CVD, KCNQ1, genetic association, Saudi population.

Received: January 11, 2017; Accepted: March 05, 2017.

\section{Introduction}

Independent genome-wide association studies (GWAS) have greatly advanced the genetics of common diseases, including cardiovascular diseases (CVD), diabetes, and cancer. Presently, over 4,800 independent loci have Hajjar reported to be associated with different diseases in various populations. Recently, a number of GWAS studies have reported a strong association between KCNQ1 (potassium voltage-gated channel, KQT-like subfamily, member 1) and type 2 diabetes mellitus (T2D). This association has been reported in various ethnic populations, including a number of European and Scandinavian countries (Unoki et al., 2008; Jonsson et al., 2009) in addition to Asian countries, specifically China (Hu et al., 2009; Liu et al., 2009; Qian et al., 2015), Korea (Lee et al., 2008), Japan (Yasuda et al., 2008), Germany (Mussig et

Send correspondence to Sadananda Acharya, Institute for Research and Medical Consultations (IRMC), University of Dammam, P. O. Box 1982, Dammam, Kingdom of Saudi Arabia - 34541. E-mail: sabaikady@uod.edu.sa. al., 2009) and India (Been et al., 2011). The KCNQ1 gene, which is located on chromosome 11q, encodes a protein that is involved in cardiac action potential. Hence, mutations in this gene are expected to influence cardiac function and are associated with the development of a number of diseases.

The Eastern Province of Saudi Arabia is known for its high prevalence of T2D and CVD. This imposes a tremendous economic and social burden on the region's population. As far as we are aware, no genetic study has been conducted on this population group with regard to the association of KCNQ1 with T2D or CVD. Therefore, the present study was conducted to determine whether KCNQ1 SNPs at loci rs151290, rs2237892 and rs2237895 are associated with T2D and CVD in the Saudi population of the Eastern Province. These particular SNPs were selected as they are more frequently reported as having an association with T2D in other populations. 


\section{Material and Methods}

\section{Study subjects}

A total of 640 Saudi patients comprising 330 individuals who had been clinically diagnosed with T2D, 250 with CVD and 60 with both T2D and CVD were recruited for the study. Additionally, the study group included 516 subjects recruited at the blood bank of King Fahd Hospital of the University after confirmation that the donors were free from T2D, CVD or any family history of these two conditions. T2D was confirmed by the definitions of the World Health Organization, 2006, while CVD cases were confirmed by a history of one or more myocardial infarction (MI). The study was approved by the University of Dammam Institutional Review Board (IRB-2013-08-026) and written informed consent was obtained from all participating subjects.

\section{Sample collection and DNA analysis}

Five milliliters of peripheral blood was collected from each participant by venipuncture into a heparinized vacutainer tube (BD Diagnostics, USA). DNA was extracted using commercially available genomic DNA purification kits (GE Healthcare, Buckinghamshire, United Kingdom). DNA concentrations and purity were measured and confirmed in a NanoDrop2000 spectrophotometer (Thermo Fisher Scientific Inc., Massachusetts, USA). The extracted DNA was stored at $-80{ }^{\circ} \mathrm{C}$ for further analysis.

\section{Genotyping}

Genotyping of the KCNQ1 SNPs, at loci rs151290, rs2237892 and rs2237895 was performed by TaqMan assay (Applied Biosystems, Foster City, CA, USA). The reactions $(25 \mu \mathrm{L})$ were run in a 96 well PCR plate on a real time PCR thermocycler (7500 Fast, ABI, Foster City, CA, USA) as per the manufacturer's recommendations. The final end-point allele calling was determined using ABI software 7500 version. 2.0.6. About $5 \%$ of the samples were run for genotyping quality assessment and were confirmed for concordance with the primary genotyping assay.

\section{Statistical analysis}

The biographic, hematological and biochemical parameters were reported as means \pm standard deviation. Intergroup significance for each variable was calculated by either Chi-square or Student's $t$-test using SPSS software. Logistic regression was used to estimate the OR (odds ratio) between the groups at $95 \% \mathrm{CI}$. Data were stratified into four clusters, namely T2D only, T2D+CVD, CVD only, and controls to analyze the association by logistic regression. All three SNPs were tested for Hardy-Weinberg equilibrium (HWE) using a Chi-square test. HaploView ver.4.2 was used to analyze linkage disequilibrium (LD) and three loci risk haplotypes (Barrett et al., 2005). Test values were considered significant when $\mathrm{p}<0.05$.

\section{Results}

The biometric parameters obtained from the patient and control individuals are given in Table 1. Allele and genotype frequencies for the SNPs tested are shown in Table 2. The male and female representation in the sample is skewed towards the male group, which may be attributed to participation bias. No significant difference in mean age among the various groups was found. There were significant differences between diabetic and non-diabetic groups for BMI and blood glucose level.

None of the KCNQ1 SNPs tested showed a statistically significant association with T2D. We further stratified the samples into T2D+CVD group for the analysis. Interestingly, comorbidity with T2D+CVD patients showed a statistically significant association for SNP rs151290 with the homozygous mutant risk genotype $(\mathrm{OR}=7.4345 ; \mathrm{p}=$ 0.0499), whereas two other SNPs (rs2237892 and rs2237895) did not show an association.

Additional recruitment of 250 more CVD patients to the sample and stratified data analysis further revealed that SNPs rs 151290 with heterozygous mutant risk genotype $(\mathrm{OR}=1.7617 ; \mathrm{p}=0.0435)$ and $\mathrm{rs} 2237895$ with homozygous mutant risk genotype $(\mathrm{OR}=2.4944 ; \mathrm{p}<0.0001)$ are strongly associated with the risk of developing CVD among the Eastern Province Saudi population.

Table 1 - Description of type 2 diabetes (T2D), cardiovascular disease (CVD) and control subjects that participated in the study.

\begin{tabular}{lcccc}
\hline Parameter & Control subjects $(\mathrm{n}=516)$ & T2D patients $(\mathrm{n}=320)$ & CVD patients $(\mathrm{n}=250)$ & T2D + CVD patients $(\mathrm{n}=60)$ \\
\hline Male $(\%)$ & $289(56.01)$ & $174(54.38)$ & $163(65.20)$ & 48 \\
Female $(\%)$ & $227(43.99) \mathrm{p}<0.0060$ & $146(45.63) \mathrm{p}=0.1175$ & $87(34.80) \mathrm{p}<0.0001$ & $12 \mathrm{p}<0.0001$ \\
Mean age (years) & $48.75 \pm 6.85$ & $51.50 \pm 8.75 \mathrm{p}<0.8875$ & $52.3 \pm 13.8 \mathrm{p}<0.8870$ & $54 \pm 9.45 \mathrm{p}<0.7783$ \\
Age range (years) & $25-65$ & $28-82$ & $32-85$ & $42-78$ \\
BMI $\left(\mathrm{kg} / \mathrm{m}^{2}\right)$ & $25 \pm 3.8$ & $27.2 \pm 4.6 \mathrm{p}<0.0001$ & $32.59 \pm 5.3 \mathrm{p}<0.0001$ & $33.47 \pm 6.5 \mathrm{p}<0.0001$ \\
Glucose $(\mathrm{mmol} / \mathrm{L})$ & $4.92 \pm 3.8$ & $8.15 \pm 4.2 \mathrm{p}<0.0001$ & $5.22 \pm 5.6 \mathrm{p}<0.3837$ & $8.27 \pm 3.6 \mathrm{p}<0.0001$ \\
\hline
\end{tabular}

BMI: body mass index. * $\mathrm{p}<0.05$ was considered significant. 


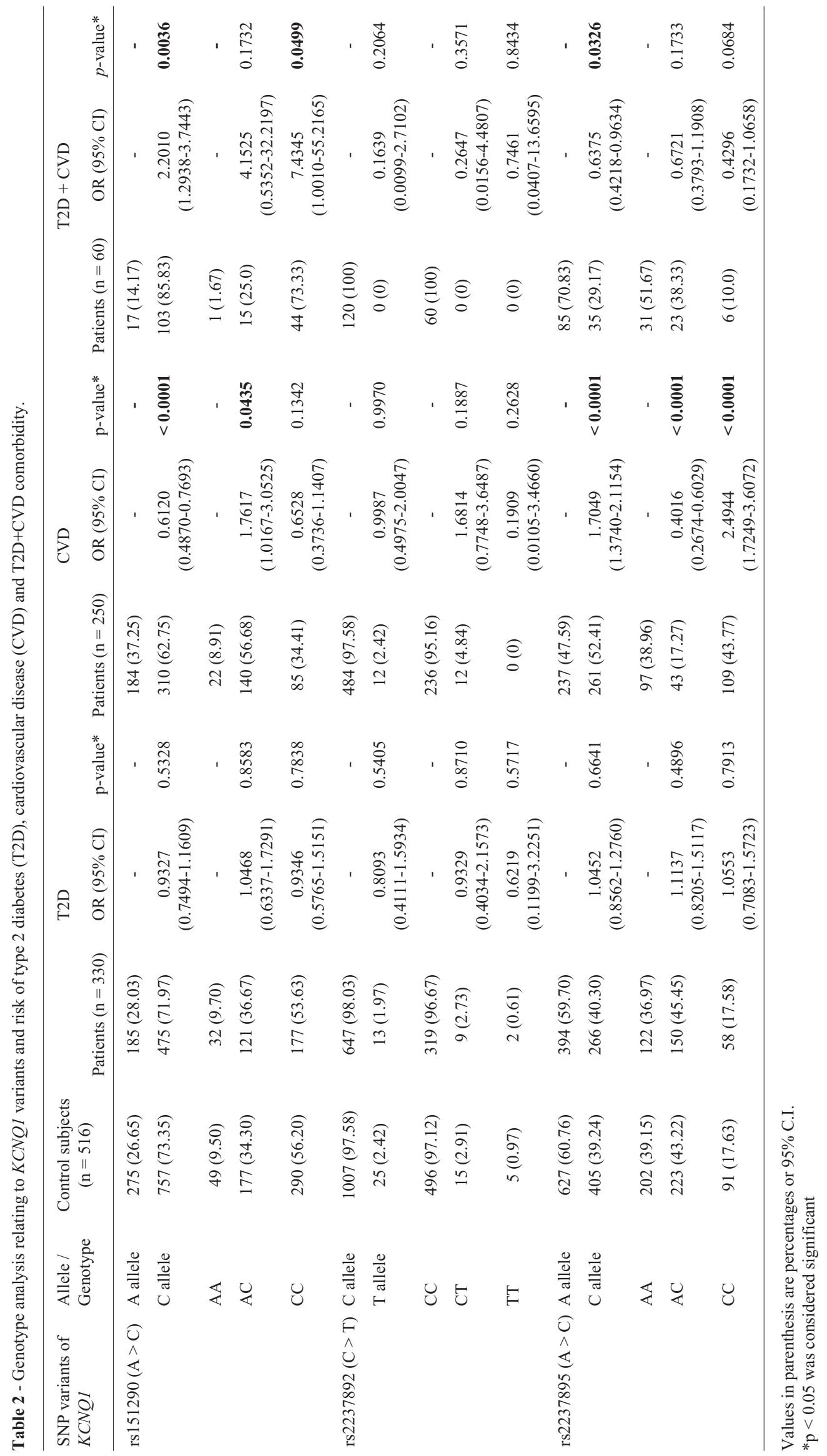


Haplotype analysis (Figure 1) revealed that ACC haplotypes (in the order of rs151290, rs2237892 and rs2237895) show the most significant risk $(\mathrm{p}<0.0001)$ allele combinations for CVD, while CCA is the most significant comorbidity risk haplotype for T2D and CVD.

\section{Discussion}

The Human Genome Project has demonstrated the important role that genetic polymorphisms play in the susceptibility and severity of both CVD and T2D. Specific polymorphisms have been recognized as being associated with an increased risk of both these diseases. Studies have identified several polymorphisms (within $K C N Q 1$ ) that are most commonly found in different populations (Lee et al., 2008; Unoki et al., 2008; Yasuda et al., 2008; Jonsson et al., 2009; Hu et al., 2009; Liu et al., 2009; Mussig et al., 2009; Been et al., 2011; Qian et al., 2015), albeit with differing frequencies. However, some reports have found a weak association between T2D and these polymorphisms.

Our study is reporting, for the first time in any population, an association between $K C N Q 1$ SNPs rs151290 and CVD. We are also reporting an association between $K C N Q 1$ SNPs rs151290 in T2D Saudi patients with CVD.
$T 2 D+C V D(n=60)$

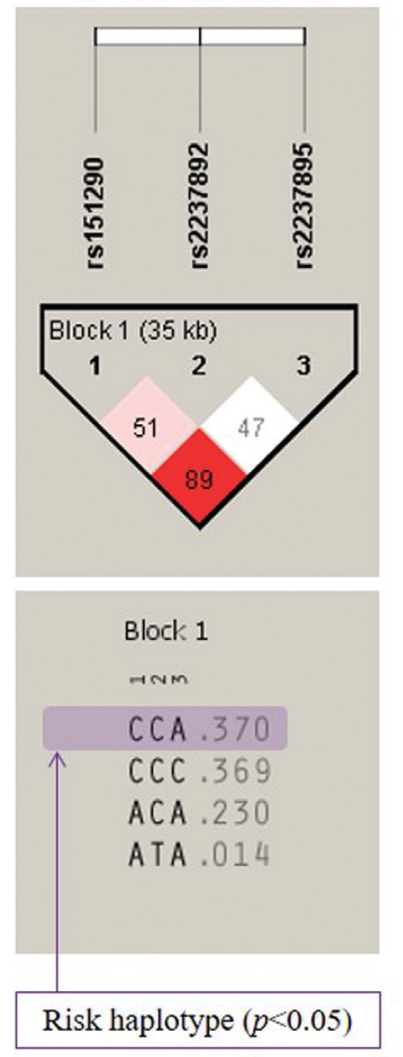

CVD $(n=250)$

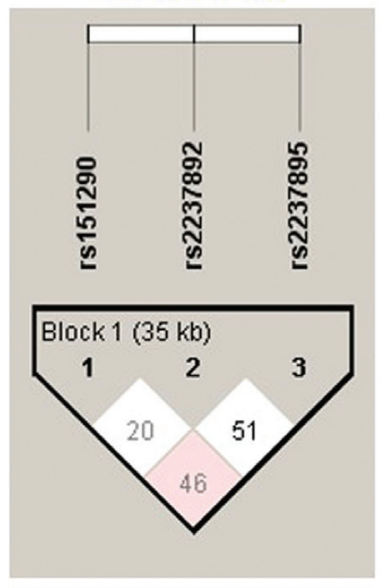

Block 1

$\rightarrow \mathrm{NMm}$

CCC.361

CCA. 324

ACA.221

ACC .070

A TA .010

Most significant risk haplotypes $(p<0.0001)$
Figure 1 - HaploView analysis of KCNQ1 SNPs; block 1 comprises SNPs rs151290, rs2237892 and rs2237895 (35kb). Red or pink block, D' (linkage disequilibrium) $<1.0$, with LOD score $>2.0$; white blocks, $\mathrm{D}^{\prime}<1.0$ with LOD score $<2.0$
There are reports that the mutant $K C N Q 1$ is implicated in various cardiac dysfunctions caused by lipid metabolism (Chen et al., 2010), familial atrial fibrillation (Bartos et al., 2013), and long QT syndrome (Hajjar and Hulot, 2014). Our findings may support those reports that these polymorphisms in $K C N Q 1$ could lead to an increased risk of developing CVD. Our data also indicate that there is no statistically significant association between these SNPs in KCNQ1 and patients with T2D. Our findings are in line with a study by Bazzi et al. (2014) that also reported a lack of association between KCNQ1 SNP rs2237892 in the Saudi population with T2D. Furthermore, a study conducted on North African Arabs also revealed a lack of association between SNPs in KCNQ1 and T2D (Turki et al., 2012). Our data contradict other studies which have suggested that these SNPs are strongly associated with T2D (Lee et al., 2008; Unoki et al., 2008; Yasuda et al., 2008; Jonsson et al., 2009; Hu et al., 2009; Liu et al., 2009; Mussig et al., 2009; Been et al., 2011; Qian et al., 2015).

Haplotype analysis of all three SNPs showed a significant risk factor for CVD [haplotype ACC: rs151290, rs2237892 and rs2237895], which confirms the allele and genotype analysis, while the CCA haplotype is a risk haplotype for T2D patients with CVD.

These results indicate that KCNQ1 polymorphism plays a significant role in the development of CVD in the Saudi population. However, the association of polymorphisms in KCNQ1 with T2D is ethnically specific and does not seem to contribute towards the development of T2D in the Saudi population. Our results implicate $K C N Q 1$ variants to be strongly associated with the risk of development of CVD, which is reported here for the first time for any population.

\section{Conclusion}

KCNQ1 gene polymorphism at loci rs151290 and rs2237895 is strongly associated with CVD, while rs 151290 is associated with comorbidity of CVD and T2D in the Saudi population of the Eastern Province. However, these variants, in addition to rs2237892, have no association with $\mathrm{T} 2 \mathrm{D}$ in this population.

\section{Acknowledgments}

This work was supported partly by the Deanship of Scientific Research (DSR), University of Dammam, grant No. 2013149 and 2014098. We thank Mr. Jonal Colendres for his laboratory and technical help during the experiments.

\section{References}

Barrett JC, Fry B, Maller J and Daly MJ (2005) Haploview: analysis and visualization of LD and haplotype maps. Bioinformatics 21:263-265. 
Bartos DC, Anderson JB, Bastiaenen R, Johnson JN, Gollob MH, Tester DJ, Burgess DE, Homfray T, Behr ER, Ackerman MJ, et al. (2013) A KCNQ1 mutation causes a high penetrance for familial atrial fibrillation. J Cardiovasc Electrophysiol 24:562-569.

Bazzi MD, Nasr FA, Alanazi MS, Alamri A, Turjoman AA, Moustafa AS, Alfadda AA, Pathan AA and Parine NR (2014) Association between FTO, MC4R, SLC30A8, and KCNQ1 gene variants and type 2 diabetes in Saudi population. Genet Mol Res 13:10194-10203.

Been LF, Ralhan S, Wander GS, Mehra NK, Singh J, Mulvihill JJ, Aston CE and Sanghera DK (2011) Variants in KCNQ1 increase type II diabetes susceptibility in South Asians: a study of 3,310 subjects from India and the US. BMC Med Genet 12:18.

Chen Z, Yin Q, Ma G and Qian Q (2010) KCNQ1 gene polymorphisms are associated with lipid parameters in a Chinese Han population. Cardiovasc Diabetol 9:35.

Hajjar RJ and Hulot JS (2014) Modeling CVD in human pluripotent cells by genome editing. J Am Coll Cardiol 64:460462.

Hu C, Wang C, Zhang R, Ma X, Wang J, Lu J, Qin W, Bao Y, Xiang K and Jia W (2009) Variations in KCNQ1 are associated with type 2 diabetes and beta cell function in a Chinese population. Diabetologia 52:1322-1325.

Jonsson A, Isomaa B, Tuomi T, Taneera J, Salehi A, Nilsson P, Groop L and Lyssenko V (2009) A variant in the KCNQ1 gene predicts future type 2 diabetes and mediates impaired insulin secretion. Diabetes 58:2409-2413.

Lee YH, Kang ES, Kim SH, Han SJ, Kim CH, Kim HJ, Ahn CW, Cha BS, Nam M, Nam CM, et al. (2008) Association between polymorphisms in SLC30A8, HHEX, CDKN2A/B,
IGF2BP2, FTO, WFS1, CDKAL1, KCNQ1 and type 2 diabetes in the Korean population. J Hum Genet 53:991-998.

Liu Y, Zhou DZ, Zhang D, Chen Z, Zhao T, Zhang Z, Ning M, Hu $X$, Yang YF, Zhang ZF, et al. (2009) Variants in KCNQ1 are associated with susceptibility to type 2 diabetes in the population of mainland China. Diabetologia 52:1315-1321.

Mussig K, Staiger H, Machicao F, Kirchhoff K, Guthoff M, Schäfer SA, Kantartzis K, Silbernagel G, Stefan N, Holst JJ, et al. (2009) Association of type 2 diabetes candidate polymorphisms in KCNQ1 with incretin and insulin secretion. Diabetes 58:1715-1720.

Qian Y, Dong M, Lu F, Li H, Jin G, Hu Z, Shen C and Shen H (2015) Joint effect of CENTD2 and KCNQ1 polymorphisms on the risk of type 2 diabetes mellitus among Chinese Han population. Mol Cell Endocrinol 407:46-51.

Turki A, Mtiraoui N, Al-Busaidi AS, Khirallah M, Mahjoub T and Almawi WY (2012) Lack of association between genetic polymorphisms within KCNQ1 locus and type 2 diabetes in Tunisian Arabs. Diabetes Res Clin Pract 98:452-458.

Unoki H, Takahashi A, Kawaguchi T, Hara K, Horikoshi M, Andersen G, Ng DP, Holmkvist J, Borch-Johnsen K, Jørgensen T, et al. (2008) SNPs in KCNQ1 are associated with susceptibility to type 2 diabetes in East Asian and European populations. Nat Genet. 40:1098-1102.

Yasuda K, Miyake K, Horikawa Y, Hara K, Osawa H, Furuta H, Hirota Y, Mori H, Jonsson A, Sato Y, et al. (2008) Variants in KCNQ1 are associated with susceptibility to type 2 diabetes mellitus. Nat Genet. 40:1092-1097.

Associate Editor: Jorge Lopez-Camelo

License information: This is an open-access article distributed under the terms of the Creative Commons Attribution License (type CC-BY), which permits unrestricted use, distribution and reproduction in any medium, provided the original article is properly cited. 